\title{
Does body height affect vascular function?
}

\author{
Shozo Yano ${ }^{1}$ \\ Received: 15 October 2021 / Accepted: 21 October 2021 / Published online: 7 December 2021 \\ (c) The Japanese Society of Hypertension 2021
}

Body height is one of the phenotypes of adulthood and is affected by both genetic and environmental factors. Interestingly, many epidemiological studies have shown that the risks of total and cardiovascular (CV) mortality were elevated in people with short stature, although no such tendency was found in some studies. According to a metaanalysis, adults with short stature showed an $\sim 1.5$ times higher risk for $\mathrm{CV}$ morbidity and mortality than tall individuals [1]. In this systematic review and meta-analysis, the authors selected 52 studies that included $>3$ million individuals. They found that among individuals within the shortest height category, the relative risks were 1.35 (95\% CI 1.25-1.44) for all-cause mortality, 1.55 (1.37-1.74) for all CV mortality, 1.49 (1.33-1.67) for coronary heart disease (CHD), and 1.52 (1.28-1.81) for myocardial infarction when compared to those within the tallest category. This finding appears to be true for both men and women, at least in the Caucasian population. In addition, a recent systematic review revealed a similar association in the Asian population [2].

Since coronary artery diameter is correlated with height in studies using angiographic measurements, physical size may contribute to the development of CHD. On the other hand, decreased vascular function is also plausible to contribute to CV mortality and morbidity. The vasculature may be damaged by perinatal exposure to poor nutrition or environmental pollutants such as tobacco, socioeconomic deprivation leading to lower-quality food and a higher prevalence of toxins, and genetic conditioning. From this point of view, Harada T. and collaborators performed a cohort study, which is published in this volume [3].

The study was performed with a total of 7682 Japanese adult men and showed that flow-mediated vasodilatation of

Shozo Yano

syano@med.shimane-u.ac.jp

1 Department of Laboratory Medicine, Shimane University Faculty of Medicine, Izumo, Japan the brachial artery (FMD) was positively associated with body height, even after adjusting for age, systolic blood pressure, current smoking, fasting blood glucose and LDL cholesterol levels, antihypertensive drug use, any lipid modification therapy, body weight, and brachial artery diameter in the multiple regression analysis [3]. FMD is related to endothelial nitric oxide bioavailability and is considered to be the gold standard for evaluating endothelial function [4]. The mean FMD values were 4.6\%, 5.2\%, $5.7 \%$, and $6.1 \%$ in the $<155.0 \mathrm{~cm}, 155.0-164.9 \mathrm{~cm}$, $165.0-174.9 \mathrm{~cm}$, and $\geq 175.0 \mathrm{~cm}$ groups, respectively. In the multiple logistic regression analysis, they found that higher height groups were significantly associated with a decreased risk of a low quartile of FMD compared with the $<155.0 \mathrm{~cm}$ group in an unadjusted model, as well as adjusted models with covariates including brachial artery diameter. According to their supplementary results, the 160.0-169.9 $\mathrm{cm}$ group and the 170.0-cm group had lower odds ratios for low quartiles of FMD than the $<160.0 \mathrm{~cm}$ group after adjustment ( 0.68 and 0.56 , respectively), suggesting that shorter Japanese men most likely have lower FMD values.

To date, we do not know the mechanism for the relationship between body height and vascular endothelial function. However, recent studies have provided us with a piece of the puzzle. Shimizu and collaborators focused on endothelial capacity to investigate the association between body height and the number of endothelial progenitor cells (CD34-positive cells) in peripheral blood samples measured by fluorescence-activated cell sorting (FACS) [5]. Bonederived hematopoietic stem cells contribute to endothelial repair and vascular maintenance. In their cross-sectional study in 231 elderly Japanese men aged 65-69 years, height was significantly associated with circulating CD34-positive cells in those with low levels of the cells but not in those with higher levels of the cells. According to their research, the circulating CD34-positive cell number was inversely associated with the cardio-ankle vascular index (CAVI), carotid intima-media thickness (IMT), active arterial wall thickening (IMT progression), and hypertension risks and positively associated with height in men with low levels of 


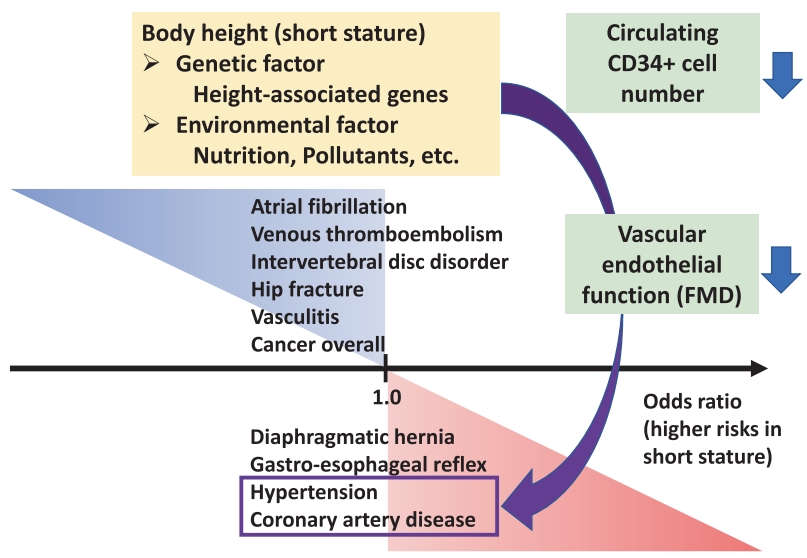

Fig. 1 Increased risk of heart disease in men with short stature and the possible mechanism. Both genetic and environmental factors are involved in body height. A recent study showed that short stature is associated with increased risks of coronary heart disease (CHD) and hypertension and associated with reduced risks of atrial fibrillation, venous thromboembolism, and cancer overall [7]. Shorter men showed a lower number of $\mathrm{CD} 34+$ cells [5] and a lower flow-mediated vasodilatation (FMD) value [3], suggesting that CD34+ cell mobilizing activity maintains vascular endothelial function, which may participate in the increased risk of CHD in men with short stature. Conversely, taller men have a lower risk of CHD

CD34-positive cells [6]. These results suggest that men with short stature tend to have decreased mobilization of CD34positive cells from bone marrow and likely have low vascular endothelial repair potential.

Body height during adulthood is associated with many disorders. According to a study using UK Biobank data, taller height was associated with reduced risks of CHD, hypertension, gastroesophageal reflux disease, and diaphragmatic hernia. Conversely, taller height was associated with increased risks of atrial fibrillation, venous thromboembolism, intervertebral disc disorder, hip fracture, vasculitis, and cancer overall [7]. Physical body size may be involved in the pathogenesis of some systemic disorders through organ structure, mechanical load, genetic factor, and environmental factors (Fig. 1). For hypertension and cardiovascular diseases, in general, body size is mostly inversely associated with heart rate, although the association between mean arterial blood pressure (MAP) and body size remains under discussion [8]. Interestingly, Poulsen et al. found a positive association between MAP and body mass in restrained animals, whereas there was no such relationship for telemetry-based MAP in freely moving animals. The difference was not likely to have been caused by the methods of restraint, including chemical or physical restraints. Thus, they cautioned that the data quality should be addressed to allow for such a discrimination to be made.
Previous cohort studies have demonstrated that body height is associated with vascular examination results, such as brachial-ankle pulse wave velocity [9], augmentation index [10], and IMT. Harada et al. added the evidence regarding FMD [3]. However, these measurements were conducted under special conditions for the examinee in a dark, quiet, temperature-controlled medical laboratory. Although no restraint is needed in human adults with normal cognition, this is not a freely moving condition. Body height or physical body mass can be attributable to environment-dependent and environment-independent vascular functions or examination results. Although vascular examination indices are comparable in one subject, we should be careful in its comparison to others. Further research, including measurement innovations of vascular function, is necessary to clarify the relationship between body height and vascular endothelial function.

\section{Compliance with ethical standards}

Conflict of interest The authors declare no competing interests.

Publisher's note Springer Nature remains neutral with regard to jurisdictional claims in published maps and institutional affiliations.

\section{References}

1. Paajanen TA, Oksala NK, Kuukasjärvi P, Karhunen PJ. Short stature is associated with coronary heart disease: a systematic review of the literature and a meta-analysis. Eur Heart J. 2010;31:1802-9.

2. Teraura H, Suzuki T, Kotani K. Association of taller stature with lower cardiovascular disease mortality in Asian people: a systematic review. J Physiol Anthropol. 2019;38:6.

3. Harada T, Kajikawa M, Maruhashi T, Kishimoto S, Yamaji T, Han Y, et al. Short stature is associated with low flow-mediated vasodilation in Japanese men. Hypertens Res. 2021 https://doi. org/10.1038/s41440-021-00785-0. Epub ahead of print.

4. Inoue T, Matsuoka H, Higashi Y, Ueda S, Sata M, Shimada KE, et al. Vascular Failure Workshop Group. Flow-mediated vasodilation as a diagnostic modality for vascular failure. Hypertens Res. 2008;31:2105-13.

5. Shimizu Y, Yamanashi H, Noguchi Y, Koyamatsu J, Nagayoshi $\mathrm{M}$, Kiyoura K, et al. Association between height and circulating CD34-positive cells taken into account for the influence of enhanced production among elderly Japanese men: a crosssectional study. Aging (Albany NY). 2019;11:663-72.

6. Shimizu Y, Maeda T. Influence of height on endothelial maintenance activity: a narrative review. Environ Health Prev Med. 2021;26:19.

7. Lai FY, Nath M, Hamby SE, Thompson JR, Nelson CP, Samani NJ. Adult height and risk of 50 diseases: a combined epidemiological and genetic analysis. BMC Med. 2018;16:187.

8. Poulsen CB, Wang T, Assersen K, Iversen NK, Damkiaer M. Does mean arterial blood pressure scale with body mass in mammals? Effects of measurement of blood pressure. Acta Physiol (Oxf). 2018;222:e13010. 
9. Andoh N, Minami J, Ishimitsu T, Ohrui M, Matsuoka H. Relationship between markers of inflammation and brachial-ankle pulse wave velocity in Japanese men. Int Heart J. 2006;47:409-20.
10. Otsuka T, Kawada T, Ibuki C, Kusama Y. Obesity as an independent influential factor for reduced radial arterial wave reflection in a middle-aged Japanese male population. Hypertens Res. 2009;32:387-91. 